\title{
Structure and floristic relationships between Cerrado sensu stricto sites on two types of substrate in northern Cerrado, Brazil
}

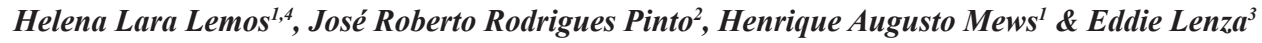 \\ ${ }^{1}$ Programa de Pós-graduação em Ciências Florestais, Universidade de Brasília - UnB, \\ CP 4357, CEP 70919-970, Brasilia, DF, Brazil \\ ${ }^{2}$ Departamento de Engenharia Florestal, Universidade de Brasília - UnB, \\ CP 4357, CEP 70919-970, Brasília, DF, Brazil \\ ${ }^{3}$ Departamento de Ciências Biológicas, Universidade do Estado de Mato Grosso - UNEMAT, \\ CP 08, CEP 78690-000, Nova Xavantina, MT, Brazil \\ ${ }^{4}$ Corresponding author: Helena Lara_Lemos, e-mail: lena_bio@yahoo.com.br
}

LEMOS, H.L., PINTO, J.R.R., MEWS, H.A. \& LENZA, E. Structure and floristic relationships between Cerrado sensu stricto sites on two types of substrate in northern Cerrado, Brazil. Biota Neotrop. (13)4: http://www.biotaneotropica.org.br/v13n4/en/abstract?article+bn02213042013

\begin{abstract}
We described and compared the floristic composition, richness, species diversity and structure of the tree-shrub component in pairs of Typical Cerrado (Cerrado Típico) and rocky outcrop Cerrado (Cerrado Rupestre) in two localities in Tocantins State. In each locality, we set up 10 plots of $20 \times 50 \mathrm{~m}$ at a site, the Cerrado Típico and other Cerrado Rupestre, and sampled the individuals with $\mathrm{Db}_{30 \mathrm{~cm}} \geq 5 \mathrm{~cm}$. The rocky outcrop Cerrado did not present any trend towards lower richness and basal area compared to the Cerrado on deep soil. Few species occurred across the four sites and only two important species (Anacardium occidentale and Qualea parviflora) in the four vegetation structure were common to both environments assessed. Furthermore, the occurrence of habitat-specialist species of rocky outcrops and high altitudes (Mimosa claussenii, Tibouchina papyrus, Schwartzia adamantium and Wunderlichia cruelsiana) and the high dissimilarity among sites suggest that altitude is the main responsible for the floristic dissimilarity, followed by the influence of substrate type. Therefore, the information with respect to phytophysiognomy type as a parameter to select areas for conservation, by itself, does not effectively ensure biodiversity preservation, owing to the existing flora heterogeneity not only at local but also at regional scale, revealed by the floristic and structural particularity of each site.
\end{abstract}

Keywords: Brazilian savanna, Cerrado Rupestre, Cerrado Típico, conservation, floristic similarity.

LEMOS, H.L., PINTO, J.R.R., MEWS, H.A. \& LENZA, E. Relações florístico-estruturais entre áreas de Cerrado sentido restrito sobre dois tipos de substrato na porção norte do Cerrado, Brasil. Biota Neotrop. 13(4): http://www.biotaneotropica.org.br/v13n4/pt/abstract?article+bn02213042013

Resumo: Descrevemos e comparamos a composição florística, a riqueza, a diversidade de espécies e a estrutura do componente arbustivo-arbóreo em pares de Cerrado Típico e Cerrado Rupestre em duas localidades no Estado de Tocantins. Em cada localidade, alocamos 10 parcelas de $20 \times 50 \mathrm{~m}$ em um sítio de Cerrado Típico e em outro de Cerrado Rupestre e amostramos os indivíduos com $\mathrm{Db}_{30 \mathrm{~cm}} \geq 5 \mathrm{~cm}$. Não identificamos tendência de que o Cerrado sobre solo raso com afloramentos rochosos tivesse riqueza e área basal inferiores ao Cerrado sobre solo profundo. Poucas espécies ocorreram nos quatro sítios e apenas duas espécies (Anacardium occidentale e Qualea parviflora) importantes para a estruturação das quatro vegetações foram comuns aos dois ambientes analisados. Aliado a isso, a ocorrência de espécies habitat-especialistas de ambientes com afloramentos rochosos e de elevadas altitudes (Mimosa claussenii, Tibouchina papyrus, Schwartzia adamantium e Wunderlichia cruelsiana) e a elevada dissimilaridade entre os sítios sugerem a altitude como principal responsável pela dissimilaridade florística, seguida pela influência do tipo de substrato. Assim, a utilização apenas da informação sobre o tipo fitofisionômico como parâmetro para escolha de áreas para conservação não garante a preservação efetiva da biodiversidade devido à heterogeneidade das floras existentes, tanto em escala local como regional, demonstrada pela particularidade florística e estrutural de cada sítio.

Palavras-chave: Savana brasileira, Cerrado Rupestre, Cerrado Típico, conservação, similaridade florística. 


\section{Introduction}

Cerrado is considered the tropical savanna with the world's greatest species richness (Silva et al. 2006), comprising nearly $33 \%$ of the Brazilian biodiversity (Aguiar et al. 2004). According to Ribeiro \& Walter (2008), the high biodiversity found in the Cerrado biome is partially due to its mosaic of plant formations as well as the different substrates on which the vegetation is established, addition of past climate changes (Oliveira-Filho \& Ratter 2002). In spite of this mosaic, the Cerrado is best characterized by the savannic formation locally named Cerrado sensu stricto, which occupies nearly $85 \%$ of the biome's core area (Eiten 1993). The Cerrado sensu stricto was subdivided by Ribeiro \& Walter (2008) into Cerrado Denso (Dense Cerrado), Cerrado Típico (Typical Cerrado), Cerrado Ralo (Sparse Cerrado) and Cerrado Rupestre (rocky outcrop Cerrado), whereas the first three occur on deep soils and the latter on shallow soils with rocky outcrops and rugged relief.

Several studies assessed phytogeographic patterns in Cerrado sensu stricto (Rizzini 1963, Warming 1973, Rizzo 1981, Felfili \& Felfili 2001, Ratter et al. 2003, Silva et al. 2006, Costa et al. 2010). Nonetheless, such studies focused on Cerrado sensu stricto sites on deep soil and mostly in the core portion of the biome, without considering the vegetation on rocky outcrops and that in peripheral, transitional or regions in contact with adjacent biomes.

Recent floristic and phytogeographic studies conducted in Cerrado sensu stricto have focused particularly on Cerrado Rupestre sites in Goiás (Lenza et al. 2011, Maracahipes et al. 2011, Santos et al. 2012) and Mato Grosso States (Felfili et al. 2002, Gomes et al. 2011) as well as in the Federal District (Amaral et al. 2006). Gomes et al. (2011) and Abreu et al. (2012) compared the Cerrado sensu stricto flora and structure on different substrates. Such authors concluded that, at local scale, the floristic and structure difference between Cerrado Rupestre and other types of Cerrado sensu stricto is reflex of the substrate on the selection of specialist species in habitats which prefer soils with rocky outcrops, dominating the community structure. Nevertheless, these types of study are very recent in other regions wherein the Cerrado sensu stricto on shallow and rocky soils occur, such as the States of Bahia, Minas Gerais and Tocantins, resulting in a gap in knowledge concerning this phytophysiognomy's flora and structure.

The fact that the Tocantins State is located in the most preserved portion of the biome (Sano et al. 2009) and in the zone of contact between Cerrado, Caatinga and Amazon Forest biomes, makes it a major source of scientific information (Instituto... 1992). This State's native vegetation presents $53.4 \%$ of its area covered by savannic and grassland formations (Cerrado sensu lato), $11.7 \%$ by forest formations (seasonal, ombrophilous and those associated with watercourses) and $34.9 \%$ by planted and natural pastures as well as anthropized areas, whereas nearly $70 \%$ of this State's terrain is suitable for agropastoral activities (Tocantins 2012). As a result, there is an inverse relation between anthropic activity expansion and knowledge regarding its vegetation diversity, floristic composition and structure. On the other hand, few studies have been conducted in the region, within which some were conducted at a single moment and presented characteristics of environmental diagnosis (Brito et al. 2002, Carvalho 2009), while others assessed the vegetation floristic and structure (Santos et al. 2006, Rezende 2007, Martins et al. 2011).

In this context, we described and compared the floristic composition, richness and diversity of species as well as the structure of the tree-shrub component of Cerrado Típico and Cerrado Rupestre pairs in two localities in Tocantins State in order to answer the following questions: 1) Are there differences in richness and diversity of tree-shrub species between adjacent Cerrado Típico and Cerrado
Rupestre sites? 2) Do both the vegetation floristic composition and structure differ between the studied sites, despite their adjacent location in the landscape? We hope that the answers to the questions can support actions aiming at conserving Cerrado's threatened biodiversity, especially its northern portion.

\section{Material and Methods}

Study areas - We selected two localities across the latitudinal gradient of Tocantins State, Brazil (Figure 1). The first locality is in the municipality of Palmas, Central portion of the State and at the margin of the Luiz Eduardo Magalhães Hydroelectric Power Plant lake, at coordinates $10^{\circ} 10^{\prime} \mathrm{S}$ and $48^{\circ} 16^{\prime} \mathrm{W}$ (Figure 1). The altitude in the region ranges from 200 to $400 \mathrm{~m}$ (Table 2) and the average annual rainfall from 1,800 to $1,900 \mathrm{~mm}$, and the main soil types are Latosols and Plinthosols (Tocantins 2012). The second location is in the municipality of Natividade, southeastern Tocantins, at coordinates $11^{\circ} 41^{\prime} \mathrm{S}$ and $47^{\circ} 42^{\prime} \mathrm{W}$ (Figure 1). The altitude ranges from 300 to $1,000 \mathrm{~m}$ (Table 2), the average annual rainfall is $1,600 \mathrm{~mm}$ and the main soil types are Latosols and Litholic, Fluvic and Quartzarenic Neosols (Tocantins 2012).

\section{Sampling}

In each locality we selected one Cerrado Típico site (Palmas $=\mathrm{TP}$ and Natividade $=\mathrm{TN})$ and one Cerrado Rupestre site $($ Palmas $=\mathrm{RP}$ and Natividade $=$ RN). In each site, we set up 10 plots of $20 \times 50 \mathrm{~m}$ and measured diameter and height of all tree-shrub individuals with base diameter measured at $30 \mathrm{~cm}$ above ground level $\left(\mathrm{Db}_{30}\right) \geq 5 \mathrm{~cm}$, as recommended by Felfili et al. (2005). We calculated the quadratic diameter of the ramifications (square root of the sum of the diameters' squares) for all individuals with forked stems below the $30 \mathrm{~cm}$, which equal the sum of the sectional areas of the branches as a descriptor of the basal area of the individual (Moro \& Martins 2011).

We used the APG III (Angiosperm... 2009) botanical classification system and the taxa names were updated from the Lista de Espécies da Flora do Brasil (Jardim... 2013). The botanical material collected will be deposited at HUTO Herbarium, University of Tocantins (UNITINS).

Data analysis - In order to compare species richness among sites we used the rarefaction method based on the number of individuals (Gotelli \& Colwell 2001) in software EcoSim 7 (Gotelli \& Entsminger $2001)$ and calculated the $p$ value based on the normal distribution $Z$ test (Zar 1999). We also performed the chi-squared test so as to investigate differences in the proportion of exclusive species and those shared among sites in software PAST 2.0 (Hammer et al. 2001). We elaborated Diversity Profiles (Tóthmérész 1995) in order to compare species diversity among sites through the Rényi exponential series in software PAST 2.0 (Hammer et al. 2001). According to Tóthmérész (1995) and Melo (2008), Diversity Profiles generalize the different weights that diversity indices provide to rare species, that is, low abundant, so that they avoid choosing one index to the detriment of the other.

In order to compare the vegetation structure among sites we assessed density and basal area per hectare, and compared diameter and height medians through Kruskal-Wallis and Mann-Whitney U test, respectively, in software PAST 2.0 (Hammer et al. 2001). In addition, we calculated the conventional phytosociological parameters (Mueller-Dombois \& Ellenberg, 1974) in software Mata Nativa 2 (CIENTEC 2006).

Furthermore, we performed the TWINSPAN (Two-Way Indicator Species Analysis) classification analysis in software PCORD 6 (McCune \& Mefford 2011) using the default cutoff level of pseudospecies $(0: 2: 5: 10: 20)$. We used this analysis to grouping 


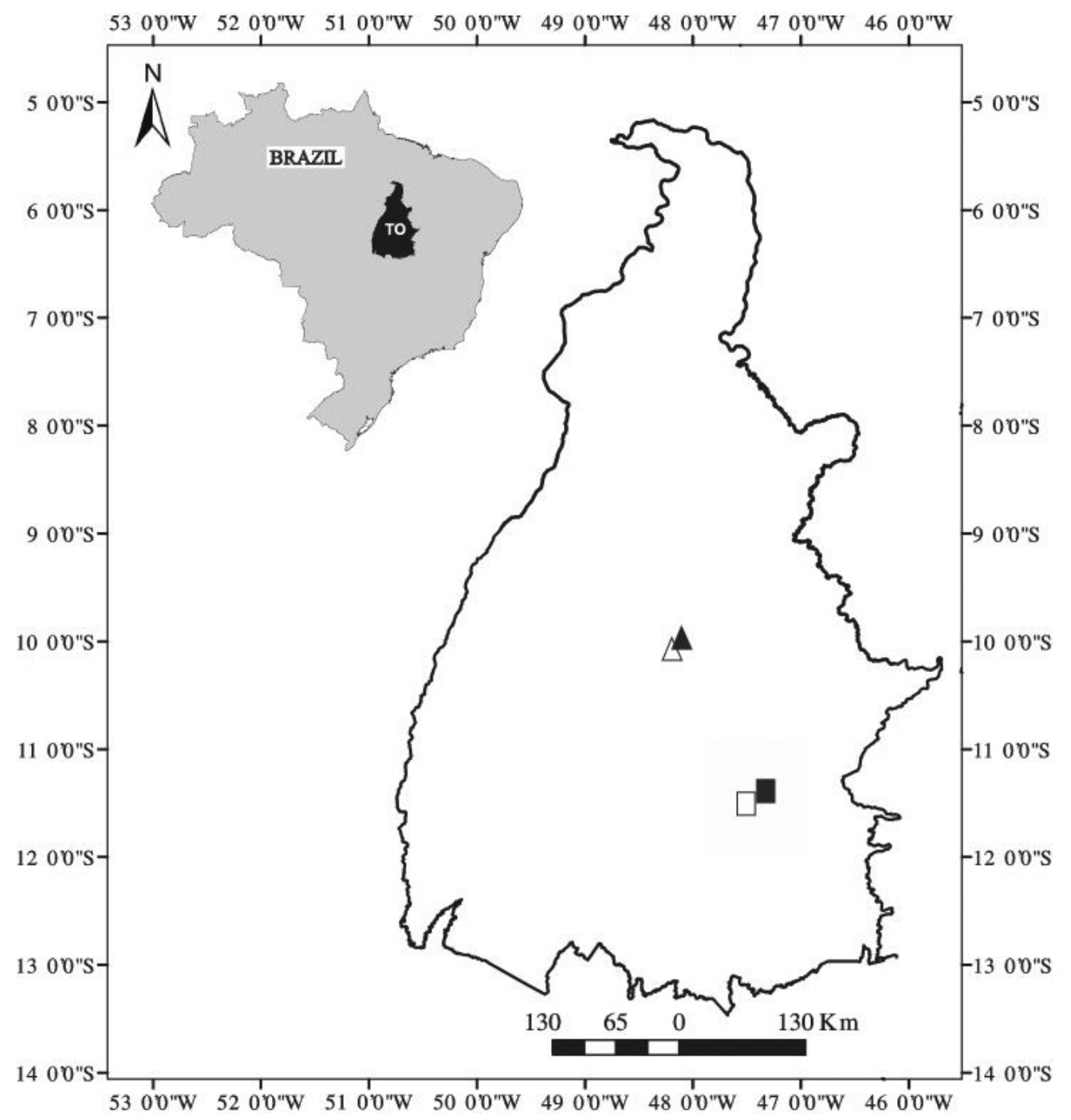

Figure 1. Locations of the Cerrado sensu stricto sites sampled on two types of substrate in Tocantins State, Brazil, pointing out the sites: Cerrado Típico Palmas

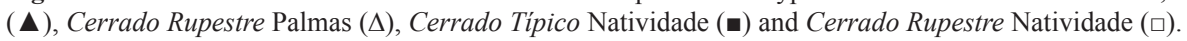

the sampling units according to floristic similarities based on species abundance in the plots (Kent \& Coker 1992). We tested the consistency of the groups formed by TWINSPAN through ANOSIM (Clarke 1993) with Bray-Curtis coefficient in software PAST 2.0 (Hammer et al. 2001). We calculated the test's significance ( $p$ ) after 9,999 permutations with sequential Bonferroni correction in the pair to pair $p$ comparison, as suggested by Quinn \& Keough (2002).

We used Jaccard similarity index (qualitative) and Bray-Curtis index (quantitative) (Magurran 2011) so as to compare the floristic composition among sites. At last, we conducted the Indicator Species Analysis (ISA) (Dufrêne \& Legendre 1997) in software PC-ORD 6 (McCune \& Mefford 2011) aiming at identifying species preference among sites. A 5\% level of significance was considered in all statistical analyses.

\section{Results}

We registered, in the four sites, 144 species distributed in 87 genera and 46 botanical families (Tables 1 and 2). We identified nine species $(6.25 \%)$ at gender level and four $(2.77 \%)$ at family level (Table 1). Within the 144 species sampled, 19 (13.19\%) were considered of wide regional distribution owing to their occurrence in the four sites, $23(15.97 \%)$ were common to three and $31(21.53 \%)$ to two sites. On the other hand, most species (71 or $49.31 \%$ ) were considered of restricted distribution since they occurred in only one site. Within the total of species registered, $24(16.7 \%)$ occurred in only one of the 40 plots sampled and, within these, 21 species $(14.6 \%)$ occurred with only one individual and were locally considered rare.

The Cerrado Típico of Natividade (TN) presented the greatest number of exclusive species, followed by RP, TP and RN (Table 2). Moreover, in TN it was also found a greater number of species, while the Cerrado Rupestre of Palmas (RP), considering its lower number of individuals, presented higher estimated and observed richness than the Cerrado Rupestre of Natividade (RN) and the Cerrado Típico of Palmas (TP).

Once the same sampling effort in terms of number of individuals (857 per area) is considered, the estimated richness in TN $\left(\mathrm{S}^{\prime}=75\right)$ was higher than RN ( $\left.\mathrm{S}^{\prime}=53 ; z=-22.00 ; p<0.0001\right)$ and $\mathrm{TP}$ $\left(\mathrm{S}^{\prime}=61 ; z=-6.26 ; p<0.0001\right)$ but lower than $\mathrm{RP}\left(\mathrm{S}^{\prime}=80 ; z=-2.24\right.$; $p=0.0127)$. In TP, 61 species would be sampled, while 80 in TP $(z=-18.12 ; p<0.0001)$ and 53 in $\mathrm{RN}(z=-7.30 ; p<0.0001)$. Yet, 53 species would be sampled in RN, which is lower than the observed richness in $\mathrm{RP}\left(\mathrm{S}^{\prime}=80 ; z=1.10 ; p<0.0001\right)$. Thus, the following decreasing order of species richness would be registered: $R P(80)>$ 
Lemos, H.L. et al.

Table 1. Tree-shrub species in Cerrado sensu stricto sites sampled on two types of substrate in Tocantins State, Brazil, and their respective phytosociological parameters. $\mathrm{RP}=$ Cerrado Rupestre Palmas; $\mathrm{RN}=$ Cerrado Rupestre Natividade; $\mathrm{TP}=$ Cerrado Típico Palmas; $\mathrm{TN}=$ Cerrado Típico Natividade; $\mathrm{D}=\mathrm{Absolute}$ Density (ind.ha $\left.{ }^{-1}\right) ; \mathrm{F}=$ Absolute Frequency (number of plots of $20 \times 50 \mathrm{~m}$, a total of 10 plots per area); DoA = Absolute Dominance in basal area $\left(\mathrm{m}^{2} . \mathrm{ha}^{-1}\right)$; IVI = Importance Value Index (\%).

\begin{tabular}{|c|c|c|c|c|c|c|c|c|c|c|c|c|c|c|c|c|}
\hline \multirow{2}{*}{ FAMILY/ SPECIES } & \multicolumn{4}{|c|}{$\mathbf{R P}$} & \multicolumn{4}{|c|}{ RN } & \multicolumn{4}{|c|}{ TP } & \multicolumn{4}{|c|}{ TN } \\
\hline & D & $\mathbf{F}$ & DoA & IVI & D & $\mathbf{F}$ & DoA & IVI & D & $\mathbf{F}$ & DoA & IVI & D & $\mathbf{F}$ & DoA & IVI \\
\hline \multicolumn{17}{|l|}{ ANACARDIACEAE } \\
\hline Anacardium occidentale L. & 25 & 9 & 0.6788 & 13.19 & 36 & 8 & 0.5770 & 13.18 & 44 & 9 & 0.2898 & 10.95 & 29 & 10 & 1.1616 & 15.31 \\
\hline Astronium fraxinifolium $\mathrm{Schott}$ & - & - & - & - & - & - & - & - & - & - & - & - & 3 & 3 & 0.0297 & 1.35 \\
\hline Tapirira guianensis Aubl. & - & - & - & - & - & - & - & - & - & - & - & - & 1 & 1 & 0.0029 & 0.39 \\
\hline \multicolumn{17}{|l|}{ ANNONACEAE } \\
\hline Annona coriacea Mart. & - & - & - & - & - & - & - & - & - & - & - & - & 1 & 1 & 0.0020 & 0.38 \\
\hline Annona crassiflora Mart. & 1 & 1 & 0.0326 & 0.83 & - & - & - & - & 2 & 2 & 0.0119 & 1.03 & - & - & - & - \\
\hline Xylopia aromatica (Lam.) Mart. & - & - & - & - & - & - & - & - & - & - & - & - & 8 & 3 & 0.0987 & 2.36 \\
\hline \multicolumn{17}{|l|}{ APOCYNACEAE } \\
\hline Aspidosperma discolor A.DC. & 1 & 1 & 0.0250 & 0.75 & - & - & - & - & - & - & - & - & - & - & - & - \\
\hline Aspidosperma macrocarpon Mart. & 4 & 3 & 0.0774 & 2.39 & 6 & 3 & 0.0398 & 2.36 & 27 & 9 & 0.1410 & 7.47 & - & - & - & - \\
\hline Aspidosperma multiflorum A.DC. & 1 & 1 & 0.0127 & 0.63 & - & - & - & - & - & - & - & - & - & - & - & - \\
\hline Aspidosperma nobile Mull.Arg. & - & - & - & - & - & - & - & - & 1 & 1 & 0.0033 & 0.48 & - & - & - & - \\
\hline Aspidosperma sp. & - & - & - & - & - & - & - & - & - & - & - & - & 1 & 1 & 0.0046 & 0.40 \\
\hline Aspidosperma tomentosum Mart. & - & - & - & - & - & - & - & - & - & - & - & - & 3 & 3 & 0.0092 & 1.17 \\
\hline Hancornia speciosa Gomes & 1 & 1 & 0.0206 & 0.71 & - & - & - & - & 21 & 8 & 0.0772 & 5.76 & 25 & 9 & 0.1209 & 5.64 \\
\hline $\begin{array}{l}\text { Himatanthus obovatus (Mull.Arg.) } \\
\text { Woodson }\end{array}$ & 1 & 1 & 0.0058 & 0.56 & 3 & 3 & 0.0162 & 1.82 & - & - & - & - & 1 & 1 & 0.0072 & 0.43 \\
\hline \multicolumn{17}{|l|}{ ARALIACEAE } \\
\hline $\begin{array}{l}\text { Schefflera vinosa (Cham. and Schltdl.) } \\
\text { Frodin and Fiaschi }\end{array}$ & 3 & 2 & 0.0272 & 1.39 & - & - & - & - & - & - & - & - & - & - & - & - \\
\hline \multicolumn{17}{|l|}{ ARECACEAE } \\
\hline Syagrus comosa (Mart.) Mart. & 13 & 6 & 0.0927 & 4.75 & - & - & - & - & - & - & - & - & 1 & 1 & 0.0054 & 0.41 \\
\hline Syagrus flexuosa (Mart.) Becc. & 5 & 4 & 0.0252 & 2.37 & - & - & - & - & - & - & - & - & 3 & 3 & 0.0123 & 1.20 \\
\hline \multicolumn{17}{|l|}{ ASTERACEAE } \\
\hline Asteraceae NI & - & - & - & - & 3 & 3 & 0.0103 & 1.76 & - & - & - & - & - & - & - & - \\
\hline Piptocarpha rotundifolia (Less.) Baker & 5 & 2 & 0.0325 & 1.68 & - & - & - & - & 4 & 2 & 0.0135 & 1.25 & - & - & - & - \\
\hline Wunderlichia cruelsiana Taub. & - & - & - & - & 9 & 6 & 0.2206 & 5.88 & - & - & - & - & - & - & - & - \\
\hline \multicolumn{17}{|l|}{ BIGNONIACEAE } \\
\hline Handroanthus ochraceus (Cham.) Mattos & 6 & 4 & 0.0724 & 2.96 & 1 & 1 & 0.0072 & 0.62 & 11 & 6 & 0.0568 & 3.84 & - & - & - & - \\
\hline $\begin{array}{l}\text { Handroanthus serratifolius (A.H.Gentry) } \\
\text { S.Grose }\end{array}$ & 3 & 3 & 0.0390 & 1.89 & - & - & - & - & - & - & - & - & - & - & - & - \\
\hline $\begin{array}{l}\text { Tabebuia aurea (Silva Manso) Benth. } \\
\text { and Hook.f. ex S.Moore }\end{array}$ & - & - & - & - & - & - & - & - & - & - & - & - & 1 & 1 & 0.0046 & 0.40 \\
\hline \multicolumn{17}{|l|}{ BURSERACEAE } \\
\hline Protium heptaphyllum (Aubl.) Marchand & - & - & - & - & - & - & - & - & - & - & - & - & 1 & 1 & 0.0025 & 0.38 \\
\hline \multicolumn{17}{|l|}{ CALOPHYLLACEAE } \\
\hline Kielmeyera coriacea Mart. and Zucc. & 2 & 2 & 0.0096 & 1.10 & 1 & 1 & 0.0026 & 0.58 & 11 & 5 & 0.0416 & 3.31 & 1 & 1 & 0.0019 & 0.38 \\
\hline Kielmeyera lathrophyton Saddi & 2 & 1 & 0.0093 & 0.71 & 5 & 2 & 0.0322 & 1.73 & 37 & 10 & 0.2730 & 10.40 & - & - & - & - \\
\hline Kielmeyera rubriflora Cambess. & 2 & 2 & 0.0064 & 1.06 & - & - & - & - & 35 & 9 & 0.1340 & 8.16 & - & - & - & - \\
\hline \multicolumn{17}{|l|}{ CARYOCARACEAE } \\
\hline $\begin{array}{l}\text { Caryocar coriaceum Wittm. } \\
\text { CELASTRACEAE }\end{array}$ & 10 & 3 & 0.5797 & 8.14 & - & - & - & - & 27 & 8 & 0.3331 & 9.46 & 23 & 9 & 0.8076 & 11.45 \\
\hline Plenckia populnea Reissek & - & - & - & - & 45 & 9 & 0.4107 & 12.81 & - & - & - & - & 2 & 2 & 0.0057 & 0.77 \\
\hline $\begin{array}{l}\text { Salacia crassifolia (Mart. Ex Schult.) } \\
\text { G.Don }\end{array}$ & 1 & 1 & 0.0127 & 0.63 & 8 & 6 & 0.0844 & 4.38 & 4 & 3 & 0.0101 & 1.55 & 8 & 4 & 0.0532 & 2.25 \\
\hline CHRYSOBALANACEAE & & & & & & & & & & & & & & & & \\
\hline $\begin{array}{l}\text { Couepia grandiflora (Mart. and Zuuc.) } \\
\text { Benth. }\end{array}$ & - & - & - & - & 51 & 9 & 0.7492 & 16.91 & - & - & - & - & 1 & 1 & 0.0072 & 0.43 \\
\hline $\begin{array}{l}\text { Hirtella ciliata Mart. and Zucc. } \\
\text { COMBRETACEAE }\end{array}$ & 4 & 2 & 0.0346 & 1.58 & - & - & - & - & 24 & 8 & 0.1030 & 6.36 & - & - & - & - \\
\hline Terminalia argentea Mart. & - & - & - & - & - & - & - & - & - & - & - & - & 1 & 1 & 0.0046 & 0.40 \\
\hline Terminalia fagifolia Mart. & - & - & - & - & 6 & 2 & 0.0732 & 2.26 & - & - & - & - & - & - & - & - \\
\hline CONNARACEAE & & & & & & & & & & & & & & & & \\
\hline Connarus suberosus Planch. & 23 & 7 & 0.1083 & 6.45 & 7 & 6 & 0.0332 & 3.75 & 12 & 5 & 0.0884 & 3.98 & 8 & 6 & 0.0342 & 2.64 \\
\hline Rourea induta Planch. & 37 & 8 & 0.1454 & 8.84 & 5 & 1 & 0.0129 & 1.09 & 2 & 2 & 0.0048 & 0.94 & 7 & 4 & 0.0239 & 1.91 \\
\hline DILLENIACEAE & & & & & & & & & & & & & & & & \\
\hline
\end{tabular}


Table 1. Continued...

\begin{tabular}{|c|c|c|c|c|c|c|c|c|c|c|c|c|c|c|c|c|}
\hline \multirow{2}{*}{ FAMILY/ SPECIES } & \multicolumn{4}{|c|}{ RP } & \multicolumn{4}{|c|}{ RN } & \multicolumn{4}{|c|}{ TP } & \multicolumn{4}{|c|}{ TN } \\
\hline & D & $\mathbf{F}$ & DoA & IVI & $\mathbf{D}$ & $\mathbf{F}$ & DoA & IVI & D & $\mathbf{F}$ & DoA & IVI & $\mathbf{D}$ & F & DoA & IVI \\
\hline Curatella americana $\mathrm{L}$. & - & - & - & - & - & - & - & - & - & - & - & - & 82 & 10 & 0.9020 & 17.43 \\
\hline Davilla grandiflora A.St.-Hil. & - & - & - & - & - & - & - & - & - & - & - & - & 2 & 2 & 0.0064 & 0.78 \\
\hline Davilla elliptica A.St.-Hil. & 65 & 8 & 0.3730 & 14.40 & - & - & - & - & 15 & 5 & 0.0504 & 3.81 & 29 & 9 & 0.1037 & 5.82 \\
\hline \multicolumn{17}{|l|}{ EBENACEAE } \\
\hline Diospyros coccolobifolia Mart. ex Miq. & 2 & 2 & 0.0166 & 1.17 & - & - & - & - & 3 & 2 & 0.0157 & 1.18 & - & - & - & - \\
\hline Diospyros hispida A.DC. & 15 & 5 & 0.1033 & 4.70 & 1 & 1 & 0.0026 & 0.58 & 3 & 2 & 0.0250 & 1.29 & 47 & 10 & 0.2874 & 9.19 \\
\hline \multicolumn{17}{|l|}{ ERYTHROXYLACEAE } \\
\hline Erythroxylum deciduum A. St.-Hil & - & - & - & - & - & - & - & - & - & - & - & - & 5 & 4 & 0.0285 & 1.78 \\
\hline Erythroxylum sp. 1 & 1 & 1 & 0.0065 & 0.57 & - & - & - & - & - & - & - & - & - & - & - & - \\
\hline Erythroxylum sp. 2 & - & - & - & - & - & - & - & - & - & - & - & - & 1 & 1 & 0.0032 & 0.39 \\
\hline Erythroxylum suberosum A. St.-Hil. & 11 & 6 & 0.0411 & 4.00 & 20 & 8 & 0.0632 & 6.26 & 8 & 3 & 0.0243 & 2.11 & 1 & 1 & 0.0024 & 0.38 \\
\hline Erythroxylum tortuosum Mart. & - & - & - & - & 1 & 1 & 0.0023 & 0.57 & - & - & - & - & 15 & 9 & 0.0474 & 4.17 \\
\hline \multicolumn{17}{|l|}{ EUPHORBIACEAE } \\
\hline Manihot sp. & - & - & - & - & - & - & - & - & 3 & 2 & 0.0076 & 1.08 & - & - & - & - \\
\hline \multicolumn{17}{|l|}{ FABACEAE } \\
\hline Andira cujabensis Benth. & 3 & 2 & 0.0917 & 2.04 & - & - & - & - & 13 & 5 & 0.1507 & 4.83 & - & - & - & - \\
\hline Andira vermifuga (Mart.) Benth. & 7 & 2 & 0.0486 & 2.07 & 7 & 5 & 0.1892 & 4.91 & 25 & 8 & 0.1552 & 7.10 & 3 & 3 & 0.0488 & 1.51 \\
\hline Bowdichia virgilioides Kunth & 5 & 3 & 0.0756 & 2.49 & 9 & 6 & 0.1931 & 5.60 & 29 & 9 & 0.2113 & 8.52 & 10 & 7 & 0.0770 & 3.46 \\
\hline Cenostigma tocantinum Ducke & - & - & - & - & - & - & - & - & 3 & 1 & 0.0077 & 0.73 & - & - & - & - \\
\hline $\begin{array}{l}\text { Chamaecrista orbiculata (Benth.) } \\
\text { H.S.Irwin and Barneby }\end{array}$ & - & - & - & - & 3 & 1 & 0.0099 & 0.85 & - & - & - & - & - & - & - & - \\
\hline Copaifera coriacea Mart. & 1 & 1 & 0.0316 & 0.82 & - & - & - & - & 10 & 3 & 0.0307 & 2.39 & - & - & - & - \\
\hline Copaifera langsdorffii Desf. & - & - & - & - & 8 & 4 & 0.1805 & 4.47 & - & - & - & - & 1 & 1 & 0.0027 & 0.39 \\
\hline Dalbergia miscolobium Benth. & - & - & - & - & - & - & - & - & 19 & 8 & 0.0883 & 5.70 & - & - & - & - \\
\hline Dimorphandra gardneriana Tul. & 13 & 6 & 0.1023 & 4.84 & - & - & - & - & 43 & 10 & 0.3231 & 11.60 & 8 & 4 & 0.0341 & 2.08 \\
\hline Dimorphandra mollis Benth. & 1 & 1 & 0.0030 & 0.53 & - & - & - & - & - & - & - & - & - & - & - & - \\
\hline Dipteryx alata Vogel & 1 & 1 & 0.0097 & 0.60 & - & - & - & - & - & - & - & - & - & - & - & - \\
\hline $\begin{array}{l}\text { Enterolobium gummiferum (Mart.) } \\
\text { J.F.Macbr. }\end{array}$ & - & - & - & - & - & - & - & - & 1 & 1 & 0.0021 & 0.47 & 1 & 1 & 0.0140 & 0.49 \\
\hline Hymenaea courbaril $\mathrm{L}$. & 1 & 1 & 0.0046 & 0.55 & - & - & - & - & - & - & - & - & - & - & - & - \\
\hline Hymenaea stigonocarpa Mart. Ex Hayne & 16 & 6 & 0.1774 & 5.95 & 2 & 2 & 0.0238 & 1.35 & - & - & - & - & 9 & 5 & 0.0538 & 2.61 \\
\hline Machaerium acutifolium Vogel & - & - & - & - & - & - & - & - & - & - & - & - & 5 & 4 & 0.0852 & 2.28 \\
\hline Machaerium opacum Vogel & - & - & - & - & 1 & 1 & 0.0024 & 0.58 & - & - & - & - & 6 & 5 & 0.1516 & 3.22 \\
\hline $\begin{array}{l}\text { Martiodendron mediterraneum (Mart. Ex } \\
\text { Benth.) R.C.Koeppen }\end{array}$ & - & - & - & - & - & - & - & - & 5 & 1 & 0.0166 & 1.04 & - & - & - & - \\
\hline Mimosa claussenii Benth. & - & - & - & - & 1 & 1 & 0.0033 & 0.59 & - & - & - & - & - & - & - & - \\
\hline Plathymenia reticulata Benth. & 3 & 2 & 0.0751 & 1.87 & - & - & - & - & 24 & 7 & 0.1356 & 6.42 & 6 & 5 & 0.0680 & 2.49 \\
\hline Pterodon emarginatus Vogel & - & - & - & - & - & - & - & - & 5 & 3 & 0.0210 & 1.78 & - & - & - & - \\
\hline Pterodon pubescens (Benth.) Benth. & - & - & - & - & 1 & 1 & 0.0424 & 0.99 & - & - & - & - & 8 & 6 & 0.1274 & 3.45 \\
\hline $\begin{array}{l}\text { Stryphnodendron adstringens (Mart.) } \\
\text { Coville }\end{array}$ & - & - & - & - & - & - & - & - & 1 & 1 & 0.0024 & 0.47 & - & - & - & - \\
\hline Stryphnodendron rotundifolium Mart. & 1 & 1 & 0.0079 & 0.58 & - & - & - & - & - & - & - & - & 2 & 2 & 0.0124 & 0.83 \\
\hline Tachigali aurea Tul. & 14 & 7 & 0.0995 & 5.32 & 33 & 5 & 0.4115 & 9.81 & 4 & 2 & 0.0167 & 1.29 & 28 & 10 & 0.2576 & 7.36 \\
\hline $\begin{array}{l}\text { Tachigali vulgaris L.G. Silva and H. C. } \\
\text { Lima }\end{array}$ & - & - & - & - & - & - & - & - & 26 & 6 & 0.1870 & 6.89 & 72 & 10 & 0.8200 & 15.89 \\
\hline Vatairea macrocarpa (Benth.) Ducke & 1 & 1 & 0.0095 & 0.60 & 2 & 2 & 0.0243 & 1.35 & 34 & 9 & 0.1351 & 8.08 & 7 & 4 & 0.0337 & 1.99 \\
\hline \multicolumn{17}{|l|}{ ICACINACEAE } \\
\hline Emmotum nitens (Benth.) Miers & 2 & 1 & 0.0269 & 0.89 & 1 & 1 & 0.0595 & 1.17 & 24 & 6 & 0.3949 & 9.23 & 1 & 1 & 0.0024 & 0.38 \\
\hline \multicolumn{17}{|l|}{ LAMIACEAE } \\
\hline Vitex polygama Cham. & 1 & 1 & 0.0147 & 0.65 & - & - & - & - & - & - & - & - & - & - & - & - \\
\hline \multicolumn{17}{|l|}{ LAURACEAE } \\
\hline Lauraceae NI & - & - & - & - & - & - & - & - & 2 & 1 & 0.0043 & 0.59 & - & - & - & - \\
\hline \multicolumn{17}{|l|}{ LOGANIACEAE } \\
\hline Strychnos pseudoquina A.St.-Hil. & 4 & 2 & 0.0711 & 1.95 & - & - & - & - & 4 & 3 & 0.0569 & 2.12 & - & - & - & - \\
\hline LYTHRACEAE & & & & & & & & & & & & & & & & \\
\hline Lafoensia pacari A.St.-Hil & 1 & 1 & 0.0115 & 0.62 & 5 & 1 & 0.0592 & 1.56 & - & - & - & - & 9 & 6 & 0.0229 & 2.62 \\
\hline MALPIGHIACEAE & & & & & & & & & & & & & & & & \\
\hline Banisteriopsis latifolia (A.Juss.) B.Gates & - & - & - & - & 18 & 6 & 0.1330 & 5.88 & - & - & - & - & - & - & - & - \\
\hline Byrsonima coccolobifolia Kunth & 2 & 2 & 0.0308 & 1.31 & 28 & 7 & 0.1764 & 7.78 & 38 & 10 & 0.1634 & 9.16 & 85 & 10 & 0.4630 & 13.86 \\
\hline
\end{tabular}


Table 1. Continued...

\begin{tabular}{|c|c|c|c|c|c|c|c|c|c|c|c|c|c|c|c|c|}
\hline \multirow{2}{*}{ FAMILY/ SPECIES } & \multicolumn{4}{|c|}{ RP } & \multicolumn{4}{|c|}{ RN } & \multicolumn{4}{|c|}{ TP } & \multicolumn{4}{|c|}{ TN } \\
\hline & D & $\mathbf{F}$ & DoA & IVI & $\mathbf{D}$ & $\mathbf{F}$ & DoA & IVI & D & $\mathbf{F}$ & DoA & IVI & $\mathbf{D}$ & $\mathbf{F}$ & DoA & IVI \\
\hline Byrsonima crassifolia (L.) Kunth & - & - & - & - & - & - & - & - & 1 & 1 & 0.0131 & 0.60 & - & - & - & - \\
\hline Byrsonima pachyphylla A.Juss. & 75 & 10 & 0.7706 & 20.33 & 35 & 5 & 0.1778 & 7.60 & 9 & 4 & 0.0311 & 2.64 & 41 & 10 & 0.2648 & 8.50 \\
\hline Byrsonima verbascifolia (L.) DC. & - & - & - & - & 2 & 1 & 0.0074 & 0.73 & 2 & 2 & 0.0052 & 0.95 & 1 & 1 & 0.0286 & 0.61 \\
\hline $\begin{array}{l}\text { Heteropterys byrsonimifolia } \text { A. Juss. } \\
\text { MALVACEAE }\end{array}$ & 25 & 6 & 0.1404 & 6.63 & 8 & 6 & 0.0476 & 4.00 & 2 & 1 & 0.0046 & 0.60 & 20 & 8 & 0.0688 & 4.49 \\
\hline $\begin{array}{l}\text { Eriotheca gracilipes (K.Schum.) A. } \\
\text { Robyns }\end{array}$ & 1 & 1 & 0.0039 & 0.54 & 26 & 7 & 0.2133 & 7.96 & 4 & 2 & 0.0434 & 1.61 & 1 & 1 & 0.0115 & 0.46 \\
\hline $\begin{array}{l}\text { Eriotheca pubescens (Mart. and Zucc.) } \\
\text { Schott and Encl. }\end{array}$ & - & - & - & - & - & - & - & - & - & - & - & - & 2 & 2 & 0.0126 & 0.84 \\
\hline Eriotheca $\mathrm{sp}$ & 6 & 4 & 0.1712 & 3.95 & - & - & - & - & - & - & - & - & - & - & - & - \\
\hline $\begin{array}{l}\text { Pseudobombax longiflorum (Mart. and } \\
\text { Zucc.) A.Robyns }\end{array}$ & - & - & - & - & - & - & - & - & - & - & - & - & 4 & 4 & 0.0341 & 1.75 \\
\hline MARCGRAVIACEAE & & & & & & & & & & & & & & & & \\
\hline $\begin{array}{l}\text { Schwartzia adamantium (Cambess.) } \\
\text { Bedell ex Gir.-Cañas }\end{array}$ & - & - & - & - & 33 & 9 & 0.8944 & 16.60 & - & - & - & - & - & - & - & - \\
\hline MELASTOMATACEAE & & & & & & & & & & & & & & & & \\
\hline Miconia albicans (Sw.) Triana & 8 & 4 & 0.0377 & 2.85 & - & - & - & - & 2 & 2 & 0.0049 & 0.94 & 2 & 2 & 0.0065 & 0.78 \\
\hline Miconia ferruginata DC. & 13 & 5 & 0.0929 & 4.37 & 8 & 2 & 0.0675 & 2.40 & - & - & - & - & 3 & 1 & 0.0364 & 0.84 \\
\hline Miconia leucarpa DC. & 1 & 1 & 0.0020 & 0.52 & - & - & - & - & - & - & - & - & - & - & - & - \\
\hline Mouriri cf. guianensis Aubl. & - & - & - & - & - & - & - & - & - & - & - & - & 3 & 3 & 0.0373 & 1.41 \\
\hline Mouriri pusa Gardner & 1 & 1 & 0.0183 & 0.68 & - & - & - & - & 31 & 9 & 1.4586 & 23.92 & - & - & - & - \\
\hline $\begin{array}{l}\text { Tibouchina papyrus (Pohl) Toledo } \\
\text { MORACEAE }\end{array}$ & - & - & - & - & 39 & 7 & 0.2072 & 9.21 & - & - & - & - & - & - & - & - \\
\hline $\begin{array}{l}\text { Brosimum gaudichaudii Trécul } \\
\text { MYRTACEAE }\end{array}$ & - & - & - & - & - & - & - & - & - & - & - & - & 4 & 3 & 0.0091 & 1.25 \\
\hline Eugenia aurata O.Berg. & - & - & - & - & 5 & 2 & 0.0232 & 1.64 & - & - & - & - & - & - & - & - \\
\hline Eugenia dysenterica DC. & - & - & - & - & - & - & - & - & - & - & - & - & 6 & 4 & 0.0377 & 1.94 \\
\hline Myrcia cf. guianensis (Aubl.) DC. & - & - & - & - & - & - & - & - & - & - & - & - & 13 & 8 & 0.1095 & 4.27 \\
\hline Myrcia cf. multiflora (Lam.) DC. & - & - & - & - & - & - & - & - & - & - & - & - & 3 & 2 & 0.0256 & 1.03 \\
\hline Myrcia cf. tomentosa (Aubl.) DC. & - & - & - & - & - & - & - & - & - & - & - & - & 5 & 3 & 0.0294 & 1.51 \\
\hline Myrcia multiflora (Lam.) DC. & 21 & 4 & 0.2477 & 6.47 & - & - & - & - & - & - & - & - & - & - & - & - \\
\hline Myrcia splendens (Sw.) DC. & 6 & 3 & 0.1247 & 3.10 & 9 & 5 & 0.0532 & 3.70 & 113 & 10 & 0.7006 & 23.06 & 9 & 3 & 0.0399 & 1.93 \\
\hline Psidium myrsinites DC. & 86 & 10 & 0.8132 & 22.04 & 10 & 4 & 0.0501 & 3.32 & - & - & - & - & 20 & 8 & 0.1129 & 4.88 \\
\hline $\begin{array}{l}\text { Psidium guianeense } \mathrm{Sw} \text {. } \\
\text { NYCTAGINACEAE }\end{array}$ & - & - & - & - & 34 & 8 & 0.1406 & 8.47 & - & - & - & - & - & - & - & - \\
\hline $\begin{array}{l}\text { Guapira graciliflora (Mart. Ex Schmidt) } \\
\text { Lundell }\end{array}$ & - & - & - & - & 3 & 3 & 0.0244 & 1.90 & 28 & 6 & 0.0931 & 5.95 & - & - & - & - \\
\hline $\begin{array}{l}\text { Neea theifera Oerst. } \\
\text { OCHNACEAE }\end{array}$ & 1 & 1 & 0.0035 & 0.54 & - & - & - & - & - & - & - & - & - & - & - & - \\
\hline Ouratea castaneifolia (DC.) Engl. & 1 & 1 & 0.0064 & 0.56 & - & - & - & - & - & - & - & - & - & - & - & - \\
\hline Ouratea riedeliana Engl. & 2 & 2 & 0.0114 & 1.11 & - & - & - & - & - & - & - & - & - & - & - & - \\
\hline Ouratea hexasperma (A.St.-Hil.) Baill. & 24 & 8 & 0.2090 & 7.97 & 5 & 2 & 0.0182 & 1.59 & 43 & 9 & 0.2242 & 10.05 & 39 & 9 & 0.1655 & 7.19 \\
\hline $\begin{array}{l}\text { Ouratea spectabilis (Mart.) Engl. } \\
\text { OLACACEAE }\end{array}$ & 4 & 4 & 0.0168 & 2.17 & - & - & - & - & - & - & - & - & - & - & - & - \\
\hline $\begin{array}{l}\text { Heisteria ovata Benth. } \\
\text { OPILIACEAE }\end{array}$ & 2 & 1 & 0.0100 & 0.72 & - & - & - & - & 7 & 4 & 0.0221 & 2.33 & - & - & - & - \\
\hline $\begin{array}{l}\text { Agonandra brasiliensis Miers ex Benth. } \\
\& \text { Hook.f. }\end{array}$ & 6 & 3 & 0.0281 & 2.13 & 1 & 1 & 0.0056 & 0.61 & 4 & 2 & 0.0098 & 1.20 & - & - & - & - \\
\hline PROTEACEAE & & & & & & & & & & & & & & & & \\
\hline $\begin{array}{l}\text { Roupala montana Aubl. } \\
\text { RUBIACEAE }\end{array}$ & 2 & 2 & 0.0184 & 1.18 & - & - & - & - & 3 & 1 & 0.0076 & 0.73 & - & - & - & - \\
\hline Chomelia ribesioides Benth. ex A.Gray & 23 & 7 & 0.2042 & 7.42 & 4 & 3 & 0.0181 & 1.94 & - & - & - & - & - & - & - & - \\
\hline Cordiera cf. elliptica (Cham.) Kuntze & - & - & - & - & 23 & 7 & 0.1164 & 6.66 & - & - & - & - & - & - & - & - \\
\hline Ferdinandusa elliptica (Pohl) Pohl & 5 & 2 & 0.1256 & 2.61 & 9 & 2 & 0.0518 & 2.34 & - & - & - & - & 29 & 8 & 0.1894 & 6.29 \\
\hline Palicourea rigida Kunth & 4 & 2 & 0.0215 & 1.45 & - & - & - & - & - & - & - & - & 2 & 2 & 0.0064 & 0.78 \\
\hline Rubiaceae 1 & 7 & 3 & 0.0364 & 2.33 & - & - & - & - & - & - & - & - & - & - & - & - \\
\hline Rubiaceae 2 & - & - & - & - & - & - & - & - & - & - & - & - & 2 & 2 & 0.0074 & 0.79 \\
\hline $\begin{array}{l}\text { Tocoyena formosa (Cham. and Schltdl.) } \\
\text { K. Schum. }\end{array}$ & 12 & 5 & 0.0586 & 3.91 & - & - & - & - & 1 & 1 & 0.0032 & 0.48 & 3 & 3 & 0.0090 & 1.17 \\
\hline
\end{tabular}


Table 1. Continued...

\begin{tabular}{|c|c|c|c|c|c|c|c|c|c|c|c|c|c|c|c|c|}
\hline \multirow{2}{*}{ FAMILY/ SPECIES } & \multicolumn{4}{|c|}{ RP } & \multicolumn{4}{|c|}{ RN } & \multicolumn{4}{|c|}{ TP } & \multicolumn{4}{|c|}{ TN } \\
\hline & D & $\mathbf{F}$ & DoA & IVI & D & $\mathbf{F}$ & DoA & IVI & D & $\mathbf{F}$ & DoA & IVI & D & $\mathbf{F}$ & DoA & IVI \\
\hline \multicolumn{17}{|l|}{ SALICACEAE } \\
\hline Casearia sylvestris Sw. & 9 & 5 & 0.0361 & 3.33 & - & - & - & - & - & - & - & - & 7 & 5 & 0.0257 & 2.20 \\
\hline \multicolumn{17}{|l|}{ SAPINDACEAE } \\
\hline Magonia pubescens A.St.-Hil. & - & - & - & - & - & - & - & - & 3 & 1 & 0.0080 & 0.74 & 6 & 4 & 0.0605 & 2.14 \\
\hline \multicolumn{17}{|l|}{ SAPOTACEAE } \\
\hline Pouteria ramiflora (Mart.) Radlk. & - & - & - & - & 8 & 2 & 0.1230 & 2.97 & 29 & 7 & 0.2475 & 8.27 & 21 & 9 & 0.1325 & 5.41 \\
\hline Pouteria sp. 1 & - & - & - & - & - & - & - & - & 2 & 2 & 0.0130 & 1.04 & - & - & - & - \\
\hline Sapotaceae 1 & 1 & 1 & 0.0027 & 0.53 & - & - & - & - & - & - & - & - & - & - & - & - \\
\hline \multicolumn{17}{|l|}{ SIMAROUBACEAE } \\
\hline \multicolumn{16}{|l|}{ SOLANACEAE } & 1.19 \\
\hline \multicolumn{16}{|l|}{ STYRACACEAE } & 0.38 \\
\hline $\begin{array}{l}\text { Styrax ferrugineus Nees and Mart. } \\
\text { SYMPLOCACEAE }\end{array}$ & \multicolumn{16}{|c|}{ SYMPLOCACEAE } \\
\hline Symplocos sp. 1 & - & - & - & - & - & - & - & - & - & - & - & - & 1 & 1 & 0.0072 & 0.43 \\
\hline \multicolumn{17}{|l|}{ URTICACEAE } \\
\hline Cecropia pachystachya Trécul & 1 & 1 & 0.0101 & 0.60 & - & - & - & - & - & - & - & - & - & - & - & - \\
\hline Cecropia sp. 1 & 1 & 1 & 0.0039 & 0.54 & - & - & - & - & - & - & - & - & - & - & - & - \\
\hline \multicolumn{17}{|l|}{ VELLOZIACEAE } \\
\hline Vellozia squamata Pohl & 3 & 1 & 0.0144 & 0.88 & 204 & 10 & 1.0357 & 35.67 & - & - & - & - & 17 & 5 & 0.0604 & 3.33 \\
\hline \multicolumn{17}{|l|}{ VOCHYSIACEAE } \\
\hline Callisthene cf. minor Mart & 17 & 5 & 0.4434 & 8.36 & 22 & 2 & 0.2534 & 5.73 & 11 & 3 & 0.0467 & 2.68 & - & - & - & - \\
\hline Callisthene major Mart and Zucc. & - & - & - & - & - & - & - & - & - & - & - & - & 91 & 8 & 0.5357 & 14.43 \\
\hline Qualea grandiflora Mart. & 16 & 4 & 0.2247 & 5.66 & - & - & - & - & 7 & 4 & 0.0927 & 3.19 & 148 & 10 & 1.5295 & 28.35 \\
\hline Qualea multiflora Mart. & - & - & - & - & - & - & - & - & - & - & - & - & 1 & 1 & 0.0042 & 0.40 \\
\hline Qualea parviflora Mart. & 94 & 10 & 1.9008 & 33.91 & 103 & 10 & 1.6009 & 31.37 & 39 & 9 & 0.6906 & 15.34 & 92 & 10 & 1.3964 & 22.57 \\
\hline Salvertia convallariodora A.St.-Hil. & 28 & 7 & 0.4524 & 10.50 & - & - & - & - & 9 & 4 & 0.2023 & 4.73 & 22 & 7 & 0.3617 & 6.93 \\
\hline Vochysia cinnamomea Pohl & - & - & - & - & - & - & - & - & 66 & 10 & 0.4719 & 15.67 & - & - & - & - \\
\hline Vochysia elliptica Mart. & - & - & - & - & - & - & - & - & - & - & - & - & 9 & 4 & 0.0370 & 2.19 \\
\hline Vochysia gardneri Warm. & 23 & 5 & 0.1598 & 6.21 & 77 & 9 & 0.6921 & 18.93 & - & - & - & - & - & - & - & - \\
\hline Vochysia rufa Mart. & 3 & 2 & 0.0170 & 1.29 & - & - & - & - & - & - & - & - & 3 & 2 & 0.0087 & 0.88 \\
\hline Vochysia thyrsoidea Pohl & - & - & - & - & - & - & - & - & - & - & - & - & 4 & 4 & 0.0122 & 1.56 \\
\hline Total & 857 & - & 9.9469 & - & 997 & - & 9.6797 & - & 1.021 & - & 8.2042 & - & 1.210 & - & 11.4828 & - \\
\hline
\end{tabular}

Table 2. Characteristics of the tree-shrub vegetation in the Cerrado sensu stricto sites sampled on two types of substrate in Tocantins State, Brazil. Alt. $=$ Minimum and maximum altitude, $\mathrm{S}=$ Number of observed species, $\mathrm{S}^{\prime}=$ Number of estimated species, $\mathrm{G}=$ genera, $\mathrm{F}=$ families, $\mathrm{D}=$ individuals, $\mathrm{AB}=$ basal area and $\mathrm{Se}=$ exclusive species to each site.

\begin{tabular}{|c|c|c|c|c|c|c|c|c|}
\hline SITES & Alt. (m) & $\mathbf{S}$ & $\mathbf{S}^{\prime}$ & $\mathbf{G}$ & $\mathbf{F}$ & D (ind.ha ${ }^{-1}$ ) & $\mathrm{AB}\left(\mathrm{m}^{2}\right)$ & Se \\
\hline Cerrado Típico Palmas (TP) & $225-238$ & 62 & $61( \pm 1.1)$ & 51 & 32 & 1,021 & 8.20 & $11(7.64 \%)$ \\
\hline Cerrado Rupestre Palmas (RP) & $374-414$ & 80 & $80( \pm 0.0)$ & 58 & 36 & 857 & 9.95 & $20(13.89 \%)$ \\
\hline Cerrado Típico Natividade (TN) & $338-351$ & 82 & $75( \pm 5.0)$ & 61 & 33 & 1,210 & 11.48 & $29(20.14 \%)$ \\
\hline Cerrado Rupestre Natividade (RN) & $491-847$ & 54 & $53( \pm 1.2)$ & 49 & 27 & 997 & 9.68 & $11(7.64 \%)$ \\
\hline Total & - & 144 & - & 87 & 46 & 4,085 & 39.31 & - \\
\hline
\end{tabular}

$\mathrm{TN}(75)>\mathrm{TP}(61)>\mathrm{RN}$ (54), evincing that there is no trend towards higher observed and estimated richness in Cerrado Típico sites compared to Cerrado Rupestre (Table 2).

The Diversity Profiles analysis reinforced the particularity of each site, since three out of four curves crossed each other (Figure 2), showing that the communities are not comparable in terms of diversity according to Tóthmérész (1995). RN was the less diverse, regardless of the diversity metric considered (Figure 2), while TN, RN and TP alternate in position with the increase in equability weight (alpha) in the diversity calculation.
Density ranged from 857 to 1,210 individuals per hectare across the sites, whereas we registered the lowest and greatest values in Cerrado Rupestre sites. Moreover, the Cerrado Típico sites had the highest value $\left(\mathrm{TN}=11.48 \mathrm{~m}^{2} \cdot \mathrm{ha}^{-1}\right)$, as well as the lowest value $\left(\mathrm{TP}=8.20 \mathrm{~m}^{2} \cdot \mathrm{ha}^{-1}\right)$ of basal area (Table 1$)$. Diameter medians were higher in Cerrado Rupestre sites than Cerrado Típico (KruskalWallis, Hc $=135.2$; $p<0.05$; Mann-Whitney test, $p<0.05)$. On the other hand, height medians were greater in Cerrado Típico sites (Kruskal-Wallis, Hc = 342.2; $p<0.05$; Mann-Whitney test, $p<0.05$ ), that is, Cerrado Rupestre plants tended to be thicker and smaller than Cerrado Típico plants. 
With respect to the phytosociological parameters, the 10 species with the highest Importance Value Indices (IVIs) accounted for $46 \%$ (TP), $54 \%(\mathrm{RP}), 59 \%(\mathrm{TN})$ and $66 \%(\mathrm{RN})$ of the total density, $63 \%$ (TP), $64 \%$ (RP), $71 \%(\mathrm{TN})$ and $69 \%(\mathrm{RN})$ of the total dominance and $31 \%(\mathrm{TP}), 30 \%(\mathrm{RP}), 27 \%(\mathrm{TN})$ and $38 \%(\mathrm{RN})$ of the frequency in the four sites. Such species, altogether, accounted for $46 \%$, $49 \%, 52 \%$ and $58 \%$ of total IVI total of the TP, RP, TN and RN, respectively (Table 1). When the 10 species with the highest IVI in $\mathrm{TP}$ and $\mathrm{TN}$ are compared to those in $\mathrm{RN}$ and $\mathrm{RP}$, we verified that only Anacardium occidentale L. and Qualea parviflora Mart. occur in both environments. Apart from Caryocar coriaceum Wittm., which occurred in three sites (TP, TN and RP), no other species within the 10 species with highest IVI occur in more than one site.

The classification analysis presented consistent results with eigenvalues higher than 0.4 . The first division separated most RN plots from the other sites (RP, TP and TN), except for the plot RN12, which remained with the RP group in the third division (Figure 3). The second division separated TP plots from the others (RP and $\mathrm{TN}$ ), except for the plot RP01, which remained with the TP group in the second division. The third division separated the TN plots from RP plots and from the plot RN12 (Figure 3). The separations

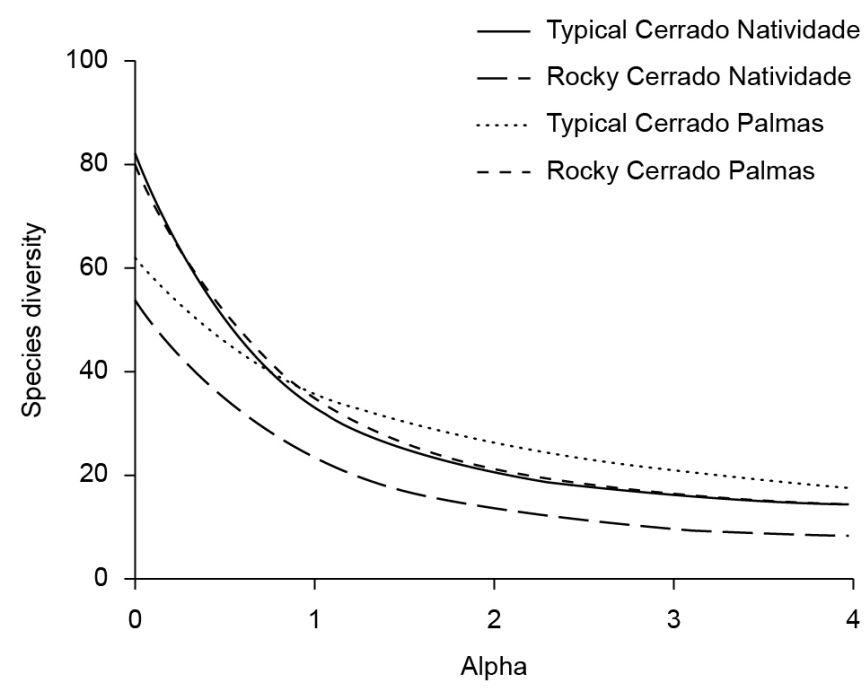

Figure 2. Diversity Profiles of tree-shrub species sampled in Cerrado sensu stricto sites on two types of substrate in Tocantins State, Brazil. by TWINSPAN analysis generated consistent groups according to ANOSIM ( $p=0.0001)$, with high dissimilarity between the groups $(R=0.90)$.

The qualitative (Jaccard) and quantitative (Bray-Curtis) similarity indices were low $(<0.45)$ between the four sites. However, TN and RP presented higher similarity to each other than to the other sites. In addition to this result, we verified that within the 144 species sampled, $66(45.8 \%)$ presented preference for TP, 12 (8\%) for RP, $19(13 \%)$ for TN and 13 (9\%) for RN (Table 3).

\section{Discussion}

The similarity in terms of richness verified among Cerrado Típico and Cerrado Rupestre sites corroborates the results of studies indicating that the Cerrado sensu stricto on deep soils does not present species richness higher than those on shallow and rocky soils (Pinto et al. 2009, Lima et al. 2010, Maracahipes et al. 2011, Abreu et al. 2012). Nevertheless, TN revealed high number of exclusive species (20.14\%), as found by Bridgewater et al. (2004) for Cerrado sensu lato. Yet the lowest number of exclusive species to $\mathrm{RN}$ and $\mathrm{TP}(7.64 \%)$ suggests that the sites with higher richness presented more exclusive species. Thereunto, our results enable us to affirm that the environmental filter represented by rocky outcrops, shallow soils and rugged relief, typical of Cerrado Rupestre, was not a limiting factor for species richness in the RP and RN sites, according to the results found by Moura et al. (2010) and Abreu et al. (2012). This supports the theory that Cerrado's environmental heterogeneity confers pattern of floristic variation to the Cerrado sensu stricto woody component (Castro \& Martins 1999, Felfili \& Felfili 2001, Durigan et al. 2003, Ratter et al. 2003, Bridgewater et al. 2004, Silva et al. 2006).

With respect to diversity, three out of four profile curves crossed each other indicating differences in richness and/or equability in these sites, which limits to determine which community presents higher or lower diversity (Tóthmérész 1995), since they vary in relation to the component values of the species diversity indices. Nonetheless, it is clear that RN presents lower diversity regardless of the diversity metric applied. This result owes to the combination of the lowest richness and low equability found in this community. We can still infer that there is similarity in terms of richness and equability among TN and RP, which presented similar diversity regardless of the diversity metric assessed. Which reflected particularity in site diversity as well as peculiarity in structure, possibly as consequence of the environmental characteristics of each site.

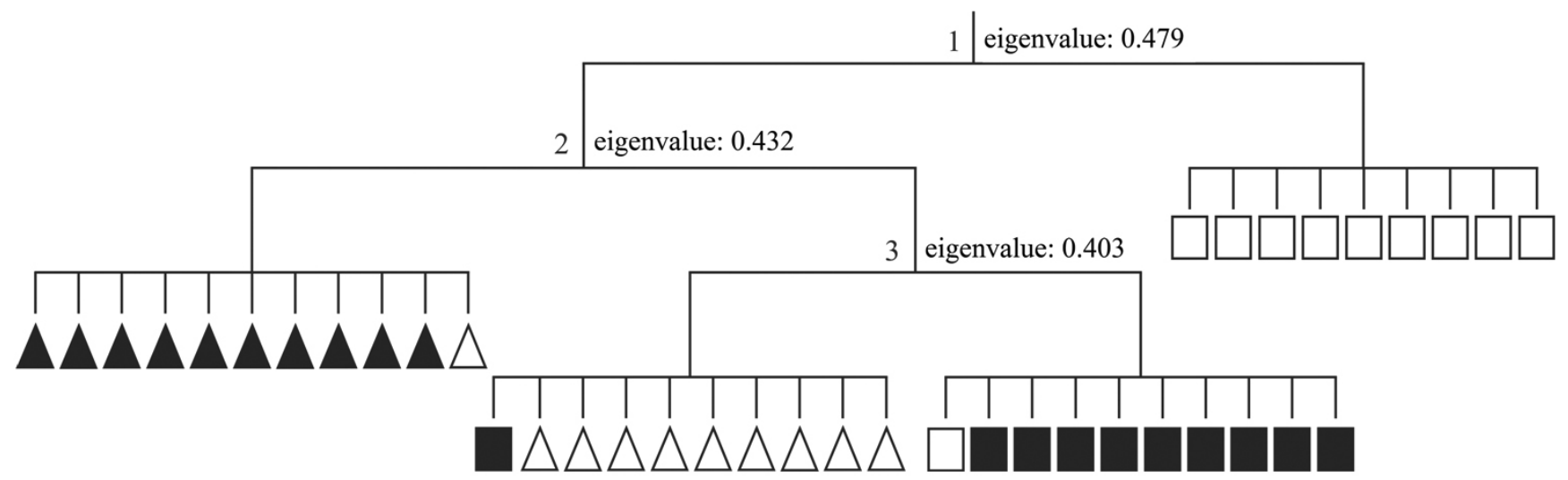

Figure 3. TWINSPAN classification of the 40 plots sampled in Cerrado sensu stricto sites on two types of substrate in Tocantins State, Brazil. Cerrado Típico

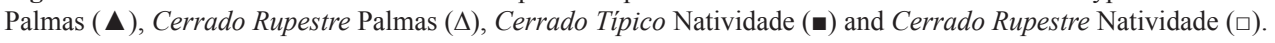


Table 3. Indicator Species Analysis (ISA) based on the abundance of tree-shrub species in the Cerrado sensu stricto sites sampled on two types of substrate in Tocantins State, Brazil. VIO = indicator value observed; VIE = indicator value estimated; $\mathrm{Sd}=$ standard deviation, $p=$ significance, $\mathrm{TP}=$ Cerrado Típico Palmas; $\mathrm{RP}=$ Cerrado Rupestre Palmas; $\mathrm{TN}=$ Cerrado Típico Natividade; $\mathrm{RN}=$ Cerrado Rupestre Natividade. The species are organized in decreasing order of VIO in each site.

\begin{tabular}{|c|c|c|c|c|c|c|c|c|}
\hline \multirow{2}{*}{ SITES/SPECIES } & \multicolumn{3}{|c|}{ VIE } & \multicolumn{5}{|l|}{ Abundance } \\
\hline & VIO & Mean & Sd & $p$ & RP & $\mathbf{R N}$ & TP & TN \\
\hline \multicolumn{9}{|l|}{ TP } \\
\hline Vochysia cinnamomea & 100.0 & 17.0 & 6.77 & 0.0002 & 0 & 0 & 100 & 0 \\
\hline Mouriri pusa & 87.2 & 17.6 & 7.17 & 0.0002 & 3 & 0 & 97 & 0 \\
\hline Kielmeyera rubriflora & 85.1 & 18.4 & 7.23 & 0.0002 & 5 & 0 & 95 & 0 \\
\hline Kielmeyera lathrophyton & 84.1 & 19.3 & 6.80 & 0.0002 & 5 & 11 & 84 & 0 \\
\hline Myrcia splendens & 82.5 & 26.3 & 7.54 & 0.0002 & 4 & 7 & 82 & 7 \\
\hline Dalbergia miscolobium & 80.0 & 17.5 & 7.98 & 0.0002 & 0 & 0 & 100 & 0 \\
\hline Vatairea macrocarpa & 69.5 & 22.9 & 7.81 & 0.0002 & 2 & 5 & 77 & 16 \\
\hline Hirtella ciliata & 68.6 & 19.1 & 7.94 & 0.0002 & 14 & 0 & 86 & 0 \\
\hline Dimorphandra gardneriana & 67.2 & 23.4 & 6.06 & 0.0002 & 20 & 0 & 67 & 12 \\
\hline Aspidosperma macrocarpon & 65.7 & 21.4 & 7.19 & 0.0002 & 11 & 16 & 73 & 0 \\
\hline Guapira graciliflora & 54.2 & 16.9 & 7.48 & 0.0012 & 0 & 10 & 90 & 0 \\
\hline Emmotum nitens & 51.4 & 16.8 & 7.25 & 0.0012 & 7 & 4 & 86 & 4 \\
\hline Plathymenia reticulata & 50.9 & 20.0 & 6.67 & 0.0010 & 9 & 0 & 73 & 18 \\
\hline Bowdichia virgilioides & 49.2 & 26.1 & 5.66 & 0.0012 & 9 & 17 & 55 & 19 \\
\hline Andira vermifuga & 47.6 & 22.4 & 6.38 & 0.0024 & 17 & 17 & 60 & 7 \\
\hline Andira cujabensis & 40.6 & 15.5 & 7.24 & 0.0098 & 19 & 0 & 81 & 0 \\
\hline Kielmeyera coriacea & 36.7 & 16.1 & 6.70 & 0.0134 & 13 & 7 & 73 & 7 \\
\hline Handroanthus ochraceus & 36.7 & 18.3 & 6.89 & 0.0266 & 33 & 6 & 61 & 0 \\
\hline Heisteria ovata & 31.1 & 13.2 & 6.69 & 0.0340 & 22 & 0 & 78 & 0 \\
\hline \multicolumn{9}{|l|}{$\mathbf{R P}$} \\
\hline Psidium myrsinites & 74.1 & 25.2 & 6.49 & 0.0002 & 74 & 9 & 0 & 17 \\
\hline Chomelia ribesioides & 59.6 & 17.4 & 7.16 & 0.0008 & 85 & 15 & 0 & 0 \\
\hline Rourea induta & 58.0 & 20.8 & 6.77 & 0.0010 & 73 & 10 & 4 & 14 \\
\hline Syagrus comosa & 55.7 & 14.7 & 6.92 & 0.0014 & 93 & 0 & 0 & 7 \\
\hline Davilla elliptica & 47.7 & 25.7 & 6.72 & 0.0078 & 60 & 0 & 14 & 27 \\
\hline Byrsonima pachyphylla & 46.9 & 30.0 & 6.30 & 0.0178 & 47 & 22 & 6 & 26 \\
\hline Myrcia multiflora & 40.0 & 12.5 & 6.91 & 0.0092 & 100 & 0 & 0 & 0 \\
\hline Ouratea spectabilis & 40.0 & 11.5 & 6.09 & 0.0098 & 100 & 0 & 0 & 0 \\
\hline Tocoyena formosa & 37.5 & 16.6 & 7.13 & 0.0254 & 75 & 0 & 6 & 19 \\
\hline Hymenaea stigonocarpa & 35.6 & 18.8 & 6.42 & 0.0248 & 59 & 7 & 0 & 33 \\
\hline Handroanthus serratifolius & 30.0 & 10.1 & 6.60 & 0.0488 & 100 & 0 & 0 & 0 \\
\hline Miconia ferruginata & 27.1 & 15.4 & 6.63 & 0.0432 & 54 & 33 & 0 & 13 \\
\hline \multicolumn{9}{|l|}{ TN } \\
\hline Curatella americana & 100.0 & 16.9 & 6.62 & 0.0002 & 0 & 0 & 0 & 100 \\
\hline Qualea grandiflora & 86.5 & 23.3 & 6.92 & 0.0002 & 9 & 0 & 4 & 87 \\
\hline Erythroxylum tortuosum & 84.4 & 17.6 & 7.35 & 0.0002 & 0 & 6 & 0 & 94 \\
\hline Callisthene major & 80.0 & 16.2 & 7.28 & 0.0002 & 0 & 0 & 0 & 100 \\
\hline Tachigali vulgaris & 73.5 & 21.2 & 6.55 & 0.0002 & 0 & 0 & 27 & 73 \\
\hline Diospyros hispida & 71.2 & 22.7 & 6.68 & 0.0002 & 23 & 2 & 5 & 71 \\
\hline Byrsonima coccolobifolia & 55.6 & 28.7 & 5.63 & 0.0006 & 1 & 18 & 25 & 56 \\
\hline Ferdinandusa elliptica & 54.0 & 18.7 & 6.79 & 0.0008 & 12 & 21 & 0 & 67 \\
\hline Pterodon pubescens & 53.3 & 14.4 & 6.77 & 0.0012 & 0 & 11 & 0 & 89 \\
\hline Hancornia speciosa & 47.9 & 22.4 & 6.48 & 0.0032 & 2 & 0 & 45 & 53 \\
\hline Machaerium opacum & 42.9 & 13.4 & 6.51 & 0.0072 & 0 & 14 & 0 & 86 \\
\hline Eugenia dysenterica & 40.0 & 11.7 & 6.30 & 0.0090 & 0 & 0 & 0 & 100 \\
\hline Erythroxylum deciduum & 40.0 & 11.8 & 6.10 & 0.0096 & 0 & 0 & 0 & 100 \\
\hline Vochysia thyrsoidea & 40.0 & 11.6 & 6.25 & 0.0098 & 0 & 0 & 0 & 100 \\
\hline Vochysia elliptica & 40.0 & 12.6 & 6.82 & 0.0106 & 0 & 0 & 0 & 100 \\
\hline Machaerium acutifolium & 40.0 & 12.0 & 6.26 & 0.0110 & 0 & 0 & 0 & 100 \\
\hline
\end{tabular}


Table 3. Continued...

\begin{tabular}{|c|c|c|c|c|c|c|c|c|}
\hline \multirow{2}{*}{ SITES/SPECIES } & \multicolumn{3}{|c|}{ VIE } & \multirow{2}{*}{$\begin{array}{c}\text { Abundance } \\
p\end{array}$} & \multirow[b]{2}{*}{$\mathbf{R P}$} & \multirow[b]{2}{*}{ RN } & \multirow[b]{2}{*}{ TP } & \multirow[b]{2}{*}{ TN } \\
\hline & VIO & Mean & Sd & & & & & \\
\hline Pseudobombax longiflorum & 40.0 & 11.6 & 6.31 & 0.0126 & 0 & 0 & 0 & 100 \\
\hline Lafoensia pacari & 36.0 & 16.2 & 7.35 & 0.0274 & 7 & 33 & 0 & 60 \\
\hline Astronium fraxinifolium & 30.0 & 10.1 & 6.54 & 0.0470 & 0 & 0 & 0 & 100 \\
\hline Brosimum gaudichaudii & 30.0 & 10.9 & 6.14 & 0.0482 & 0 & 0 & 0 & 100 \\
\hline Aspidosperma tomentosum & 30.0 & 10.1 & 6.60 & 0.0486 & 0 & 0 & 0 & 100 \\
\hline Magonia pubescens & 26.7 & 12.7 & 6.44 & 0.0368 & 0 & 0 & 33 & 67 \\
\hline \multicolumn{9}{|l|}{$\mathbf{R N}$} \\
\hline Vellozia squamata & 91.1 & 22.9 & 7.85 & 0.0002 & 1 & 91 & 0 & 8 \\
\hline Schwartzia adamantium & 90.0 & 15.9 & 6.48 & 0.0002 & 0 & 100 & 0 & 0 \\
\hline Couepia grandiflora & 88.3 & 16.8 & 6.54 & 0.0002 & 0 & 98 & 0 & 2 \\
\hline Plenckia populnea & 86.2 & 18.8 & 7.46 & 0.0002 & 0 & 96 & 0 & 4 \\
\hline Psidium guianeense & 80.0 & 15.4 & 6.78 & 0.0002 & 0 & 100 & 0 & 0 \\
\hline Cordiera cf. elliptica & 70.0 & 14.6 & 6.73 & 0.0002 & 0 & 100 & 0 & 0 \\
\hline Tibouchina papyrus & 70.0 & 14.4 & 6.57 & 0.0002 & 0 & 100 & 0 & 0 \\
\hline Vochysia gardneri & 69.3 & 19.7 & 6.46 & 0.0002 & 23 & 77 & 0 & 0 \\
\hline Banisteriopsis latifolia & 60.0 & 14.0 & 6.75 & 0.0004 & 0 & 100 & 0 & 0 \\
\hline Wunderlichia cruelsiana & 60.0 & 13.3 & 6.43 & 0.0006 & 0 & 100 & 0 & 0 \\
\hline Eriotheca gracilipes & 56.9 & 19.2 & 7.78 & 0.0022 & 3 & 81 & 13 & 3 \\
\hline Erythroxylum suberosum & 40.0 & 22.5 & 6.49 & 0.0146 & 28 & 50 & 20 & 3 \\
\hline Copaifera langsdorffii & 35.6 & 13.2 & 6.82 & 0.0244 & 0 & 89 & 0 & 11 \\
\hline
\end{tabular}

The highest density found in Cerrado Típico and the lowest in Cerrado Rupestre, and the lack of this trend in basal area corroborate the assertion that rocky outcrops and incipient soils, apparently limiting to the establishment of tree-shrub vegetation (Ribeiro \& Walter 2008), did not act as barriers against development in terms of basal area. However, Lima et al. (2010) and Lenza et al. (2011) found similar density and basal area between Cerrado Rupestre and Cerrado Típico sites, showing the lack of clear pattern of separation between these two phytophysiognomy subtypes based on density and basal area. Nevertheless, the higher median diameter values and the lower height values of individuals in the Cerrado Rupestre sites allow us to assume that the substrate limits development in height but not in basal area.

The structural importance of Qualea parviflora Mart. and Anacardium occidentale L. registered in the four sites, both of wide distribution in the Cerrado biome (Ratter et al. 2003), was also pointed out by Gomes et al. (2011) as common and important species in the vegetation structure of Cerrado on deep and shallow soils with rocky outcrops in east of Mato Grosso State. Caryocar coriaceum Wittm. was important in terms of IVI in RP, TP and TN, which corroborates the assertion that this species is indicator of the North-Northeastern Group flora, as classified by Ratter et al. (2003), present in the transition between Cerrado and Caatinga. The difference in high-IVI species composition between the four sites, with two to three species in common, was strengthened by the high floristic dissimilarity between the sites, which can be a reflex of environmental particularities. The influence of the adjacent biomes, Caatinga and Amazonia (Felfili et al. 2002, Lenza et al. 2011), also reinforces the floristic and structural particularity of the sites with occurrence of Dimorphandra gardneriana Tul. (Castro et al. 1998) and Cenostigma tocantinum Ducke in TP, considering that Tocantins State is located in a transition zone between three major biomes: Amazonia, Cerrado and Caatinga (Instituto... 1992).

Our results corroborate Felfili \& Felfili (2001), who observed that adjacent Cerrado sensu stricto sites in different conditions of substrate present reduced similarity. This can be explained by the fact that, at local scale, the physical-chemical properties of the soils drive the floristic differentiation of the tree-shrub vegetation between the Cerrado Rupestre and Cerrado Típico sites (Abreu et al. 2012). Moreover, the distance between sites does not seem to influence much on their similarity, since not only those of the same phytophysiognomy type distant from each other (RN and RP), but also those of different phytophysiognomy types adjacent to each other (TN and RN) were dissimilar.

RN separation from the other sites, including RP, on similar substrate conditions may be related to altitude, which is considered one of the responsible factors for Cerrado's floristic patterns (Munhoz \& Proença 1998, Castro \& Martins 1999, Ratter et al. 2003, Bridgewater et al. 2004, Lenza et al. 2011). The preference of specialist species to rocky outcrop and high altitude habitat registered in RN (> 400 m.a.s.1.), such as Mimosa claussenii, Tibouchina papyrus, Schwartzia adamantium and Wunderlichia cruelsiana (Ratter et al. 2000, Ribeiro \& Walter 2008, Pinto et al. 2009), whereas the last three were present within the most important ones in the vegetation structure, increase the floristic particularity of the Cerrado Rupestre sampled in Natividade. On the other hand, the absence of these species in RP $(<400$ m.a.s.l.) evinces the influence of altitude on Cerrado Rupestre's floristic composition and corroborates the results found by Gomes et al. (2011).

The separation of the other sites can be related to local edaphic factors since TP, on more sandy soil, was separated from the others, presenting Vochysia cinnamomea Pohl, normally associated with sandy environments (Finger 2008), as one of the 10 most important species in the community structure. Most of TP's preferential species indicated by ISA are frequent in Cerrado sensu stricto; however, species such as Hirtella ciliata Mart. and Zucc., whose distribution is restricted to the northeast region of the biome (Ratter et al. 2000), reveal the influence of adjacent biomes on the floristic composition of this site. The rocky outcrop was most likely responsible for separating TN and RP. Moreover, ISA's indication of Astronium fraxinifolium 
Schott (Bridgewater et al. 2004), mainly found on mesotrophic soils (more fertile), and Magonia pubescens A.St.-Hil, indicator of fertile soils (Ratter et al. 2003), as preferential in TN suggests that this site presents more fertile soils. Our results corroborate the affirmation that local environmental characteristics are responsible for Cerrado's mosaic of vegetation and for the species distribution (Ratter et al. 2000). The non-formation of groups with similar characteristics such as presence of rocky outcrops or related to geographical proximity indicates that environmental peculiarities of each location influence on the floristic composition and structure of these communities.

At last, despite the similarity in species richness among the phytophysiognomies and the representativeness of this richness in terms of Cerrado biome, we did not register trend in Cerrado Típico sites towards higher tree-shrub species richness and diversity compared to Cerrado Rupestre sites. The floristic particularity of each site was evinced by the high number of species of limited geographic distribution, the low number of species shared among sites and by only two species in common, emphasizing $\mathrm{RN}$, wherein it was found species considered indicator of rocky environments. Therefore, the information with regard to phytophysiognomy type as a parameter to select areas for conservation, by itself, does not effectively ensure biodiversity preservation, owing to the existing flora heterogeneity not only at local but also at regional scale, revealed by the floristic and structural particularity of each site.

\section{Acknowledgments}

We thank the Coordenação de Aperfeiçoamento de Pessoal de Nível Superior (CAPES) for granting a scholarship to H.L. Lemos and H.A. Mews. The Conselho Nacional de Desenvolvimento Científico e Tecnológico (CNPq) for granting the Research Productivity fellowship (PQ) to J.R.R. Pinto. The Decanato de Pesquisa e PósGraduação of the Universidade de Brasília (UnB) for partially funding the field work. The botanical experts Carolyn E.B. Proença, Manoel Cláudio da Silva-Júnior and Jair E. Q. de Faria Júnior for assisting us in the botanical material identification. And we also thank those who helped us in the field work: Tiago A. Araújo, Isadora C. Alvarez, Ana C. F. Domingos, Enderson A. Nunes, Hugo V. M. Parente and Hercules Santana.

\section{References}

ABREU, M.F., PINTO, J.R.R., MARACAHIPES, L., GOMES, L., OLIVEIRA, E.A., MARIMON, B.S., MARIMON-JÚNIOR, B.H., FARIAS, J. \& LENZA, E. 2012. Influence of edaphic variables on the floristic composition and structure of the tree-shrub vegetation in typical and rocky outcrop cerrado areas in Serra Negra, Goiás State, Brazil. Braz. J. Bot. 35(3):259-272. http://dx.doi.org/10.1590/S180699592012000300005

AGUIAR, L.M.S., MACHADO, B.M. \& MARINHO-FILHO, J.A. 2004 Diversidade Biológica do Cerrado. In Cerrado: ecologia e caracterização (L.M.S. Aguiar \& A.J.A. Camargo, eds.). Embrapa Cerrados, Planaltina, p.17-40.

AMARAl, A.G., PEREIRA, F.F.O. \& MUNHOZ, C.B.R. 2006. Fitossociologia de uma área de cerrado rupestre na Fazenda Sucupira, Brasília-DF. Cerne 12(4):350-359.

ANGIOSPERM PHYLOGENY GROUP III - APG III. 2009. An update of the Angiosperm Phylogeny Group classification for the orders and families of flowering plants: APG III. Bot. J. Linn. Soc. 161(2):105-121. http:// dx.doi.org/10.1111/j.1095-8339.2009.00996.x

BRIDGEWATER, S., RATTER, J.A. \& RIBEIRO, J.F. 2004 Biogeographic patterns, b-diversity and dominance in the cerrado biome of Brazil. Biodivers. Conserv. 13(12):2295-2318. http://dx.doi.org/10.1023/ B:BIOC.0000047903.37608.4c
BRITO, E.R., SILVA, E., MARTINS, S.V. \& RIBEIRO, G.A. 2002. Perfil ambiental do empreendimento denominado de "praias fluviais", Estado do Tocantins. Rev. Árvore 26(3):349-355. http://dx.doi.org/10.1590/ S0100-67622002000300010

CARVALHO, T.M. 2009. Síntese de campo do trecho Peixe a Ipueiras, rio Tocantins : uma contribuição à Exploratória Rio Tocantins. Rev. Esp. Acad. 8:1-6.

CASTRO, A.A.J.F. \& MARTINS, F.R. 1999. Cerrados do Brasil e do Nordeste: caracterização, área de ocupação e considerações sobre a sua fitodiversidade. Pesqui. Foco 7(9):147-178.

CASTRO, A.A.J.F., MARTINS, F.R. \& FERNANDES, A.G. 1998. The woody flora of cerrado vegetation in the state of Piauí, Northeastern Brazil. Edinb. J. Bot. 55(3):455-472. http://dx.doi.org/10.1017/S0960428600003292

CIENTEC. 2006. Mata Nativa 2: Sistema para a Análise Fitossociológica e elaboração de Inventários e Planos de Manejo de Florestas nativas. MGCIENTEC - Consultoria de Desenvolvimento de Sistemas LTDA, Viçosa.

CLARKE, K.R.1993. Non-parametric multivariate analysis of changes in community structure. Australian. J. Ecol. 18(1):117-143. http://dx.doi. org/10.1111/j.1442-9993.1993.tb00438.x

COSTA, F.V., OLIVEIRA, K.N., NUNES, Y.R.F., MENINO, G.C.O., BRANDÃO, D.O., ARAÚJO, L.S., MIRANDA, W.O. \& D'ÂNGELO NETO, S. 2010. Florística e estrutura da comunidade arbórea de duas áreas de cerrado sentido restrito no norte de Minas Gerais. Cerne 16(3):267-281.

DUFRÊNE, M. \& LEGENDRE, P. 1997. Species assemblages and indicator species: the need for flexible asymmetrical approach. Ecol. Monogr. 67(3):345-366.

DURIGAN, G., RATTER, J.A. \& BRIDGEWATER, S. 2003. Padrões fitogeográficos do cerrado paulista sob uma perspectiva regional. Hoehnea 30(1):39-51.

EITEN, G. 1993. Vegetação do Cerrado. In Cerrado: caracterização, ocupação e perspectivas. (M.N. Pinto, ed.). 2. ed. EdUnB, Secretaria do Meio Ambiente, Ciência e Tecnologia, Brasília, p.17-74.

FELFILI, M.C. \& FELFILI, J.M. 2001. Diversidade Alfa e Beta no Cerrado sensu stricto da Chapada Pratinha, Brasil. Acta Bot. Bras. 15(2):243-254. http://dx.doi.org/10.1590/S0102-33062001000200010

FELFILI, J.M., NOGUEIRA,P. E., SILVA-JÚNIOR, M.C., MARIMON, B.S. \& DELITTI, W.B.C. 2002. Composição florística e fitossociológica do Cerrado sentido restrito no município de Água Boa-MT. Acta Bot. Bras. 6(1):103-112.

FELFILI, J.M., CARVALHO, F.A. \& HAIDAR, R.F. 2005. Manual para o monitoramento de parcelas permanentes nos biomas Cerrado e Pantanal Universidade de Brasília, Departamento de Engenharia Florestal, Brasília, $55 \mathrm{p}$.

FINGER, Z. 2008. Fitossociologia de comunidades arbóreas em savanas no Brasil Central. Tese de Doutorado. Universidade Federal de Santa Maria, Santa Maria.

GOMES, L., LENZA, E., MARACAHIPES, L., MARIMON, B.S. \& OLIVEIRA, E.A. 2011. Comparações florísticas e estruturais entre duas comunidades lenhosas de cerrado típico e cerrado rupestre, Mato Grosso, Brasil. Acta Bot. Bras. 25(4):865-875. http://dx.doi.org/10.1590/S010233062011000400013

GOTELLI, N.J. \& COLWELL, A.M. 2001. Quantifying biodiversity: procedures and pitfalls in the measurement and comparison of species richness. Ecol. Lett. 4(4):379-391. http://dx.doi.org/10.1046/j.14610248.2001.00230.x

GOTELLI, N.J. \& ENTSMINGER, G.L. 2001. EcoSim: Null models software for ecology. Acquired Intelligence Inc. \& Kesey-Bear. http://homepages. together.net/ gentsmin/ecosim.htm.

HAMMER, O., HARPER, A.T.D \& RYAN, P.D. 2001. PAST: Paleontological Statistics Software Package for Education and data analysis. Paleontol. Electron. 4(1):1-9.

INSTITUTO BRASILEIRO DE GEOGRAFIA E ESTATÍSTICA - IBGE. 1992. Manual Técnico da Vegetação Brasileira. IBGE, Departamento de Recursos Naturais e Estudos Ambientais, Rio de Janeiro, 92p. 
Lemos, H.L. et al.

JARDIM BOTÂNICO DO RIO DE JANEIRO. Lista de Espécies da Flora do Brasil. 2013. http://floradobrasiljbrj.gov.br (último acesso em 20/04/2013)

KENT, M. \& COKER, P. 1992. Vegetation description and analyses, a pratical approach. Behaven Press, London, 363p.

LENZA, E., PINTO, J.R.R., MARACAHIPES, L. \& BRUZIGUESSI, E.P. 2011. Comparação da vegetação arbustivo-arbórea de uma área de cerrado rupestre na Chapada dos Veadeiros, Goiás, e áreas de cerrado sentido restrito do Bioma Cerrado. Rev. Bras. Bot. 34(3):247-259. http://dx.doi. org/10.1590/S0100-84042011000300002

LIMA, A.L., PINTO, J.R.R., LENZA, E. \& PINTO, A.S. 2010. Florística e estrutura da vegetação arbustivo-arbórea em uma área de cerrado rupestre no Parque Estadual da Serra de Caldas Novas, Goiás. Biot. Neotrop. 10(2):159-166 http://www.biotaneotropica.org.br/v11n1/en/ fullpaper?bn02111012011 (último acesso em 05/04/2013)

MAGURRAN, A.E. 2011. Medindo a diversidade biológica. Editora UFPR, Curitiba, 256p.

MARACAHIPES, L., LENZA, E., MARIMON, B.S., OLIVEIRA, E.A., PINTO, J.R.R. \& MARIMON-JÚNIOR, B.H. 2011. Estrutura e composição florística da vegetação lenhosa em cerrado rupestre na transição Cerrado-Floresta Amazônica, Mato Grosso, Brasil. Biot. Neotrop. 11(1):133-142 http://www.biotaneotropica.org.br/v11n1/en/ fullpaper?bn02111012011 (last acess in 03/04/2013)

MARTINS, S.V., BRITO-IBRAHIM, E.R., EISENLOHR, P.V., OLIVEIRAFILHO, A.T. \& SILVA, A.F. 2011. A vegetação de Ipucas no Tocantins: estudo de caso e relações florísticas com remanescentes do Cerrado e da Amazônia. In: Fitossociologia no Brasil: métodos e estudos de caso (J.M. Felfili, P.V. Eisenlohr, M.M.R.F. Melo, L.A. Andrade \& J.A.A. Meira Neto, eds.). Editora UFV, Viçosa, p.460-478.

McCUNE, B. \& MEFFORD, M.J. 2011. PC-ORD: Multivariate Analysis of Ecological Data. MjM Software Design, Gleneden Beach, Oregon.

MELO, A.S. 2008. O que ganhamos 'confundindo' riqueza de espécies e equabilidade em um índice de diversidade? Biot. Neotrop. 8(3):2127. http://www.biotaneotropica.org.br/v8n3/en/abstract?point-ofview+bn00108032008 (last acess in 02/03/2013)

MORO, M.F. \& MARTINS, F.R. 2011. Métodos de Levantamentos do Componente Arbóreo-Arbustivo. In: Fitossociologia no Brasil: métodos e estudos de caso (J.M. Felfili, P.V. Eisenlohr, M.M.R.F. Melo, L.A. Andrade \& J.A.A. Meira Neto, eds.). Editora UFV, Viçosa, p.174-212.

MOURA, I.O., GOMES-KLEIN, V.L., FELFILI, J.M. \& FERREIRA, H.D. 2010. Diversidade e estrutura comunitária de cerrado sensu stricto em afloramentos rochosos no parque estadual dos Pireneus, Goiás. Rev. Bras. Bot. 33(3):455-467. http://dx.doi.org/10.1590/S010084042010000300008

MUELLER-DOMBOIS, D. \& ELLENBERG, H. 1974. Aims and methods of vegetation ecology. John Wiley and Sons, New York, 547p.

MUNHOZ, C.B.R. \& PROENÇA, C.E.B. 1998. Composição florística do município de Alto Paraíso de Goiás na Chapada dos Veadeiros. Bol. Herb. Ezequias Paulo Heringer 3:102-150.

OLIVEIRA-FILHO, A.T. \& RATTER, J.A. 2002. Vegetation Physiognomies and Woody Flora of the Cerrado Biome. In: The Cerrados of Brazil (P.S. Oliveira \& R.J. Marquis, eds.). Columbia University Press, New York, p.91-120.
PINTO, J.R.R., LENZA, E. \& PINTO, A.S. 2009. Composição florística e estrutura da vegetação arbustivo-arbórea em um cerrado rupestre, Cocalzinho de Goiás, Goiás. Rev. Bras. Bot. 32(1):23-32. http://dx.doi. org/10.1590/S0100-84042009000100002

QUINN, G.P. \& KEOUGH, M.J. 2002. Experimental design and data analysis for biologists. Cambridge University Press, Cambridge, 537p. http:// dx.doi.org/10.1017/CBO9780511806384

RATTER, J.A., BRIDGEWATER, S., RIBEIRO, J.F., DIAS, T.A.B. \& SILVA, M.R. 2000. Estudo preliminar da distribuição das espécies lenhosas da fitofisionomia cerrado sentido restrito nos estados compreendidos pelo bioma Cerrado. Bol. Herb. Ezechias Paulo Heringer 5:5-43.

RATTER, J.A., BRIDGEWATER, S. \& RIBEIRO, J.F. 2003. Analysis of the floristic composition of the Brazilian Cerrado vegetation III: comparison of the woody vegetation of 376 areas. Edinb. J. Bot. 60(1):57-109. http:// dx.doi.org/10.1017/S0960428603000064

REZENDE, J.M. 2007. Florística, Fitossociologia e influência do gradiente de umidade do solo em campos limpos úmidos no Parque Estadual do Jalapão, Tocantins. Dissertação de Mestrado, Universidade de Brasília, Brasília.

RIBEIRO, J.F. \& WALTER, B.M. 2008. As Principais Fitofisionomias do Bioma Cerrado. In Cerrado: ecologia e flora. (S. M. Sano, S.P. Almeida \& J.F. Ribeiro, eds.). 2. ed. Embrapa Informação Tecnológica, Brasília, p.151-212.

RIZZINI, C.T. 1963. A flora do Cerrado: análise florística das savanas centrais. Simpósio sobre o Cerrado. Edgard Blucher, EDUSP, São Paulo.

RIZZO, J.A. 1981. A Flora do Estado de Goiás: coleção Rizzo. Universidade Federal de Goiás, Goiânia.

SANO, E.E., ROSA, R., BRITO, J.L.S., FERREIRA, L.G. \& BEZERRA, H.S. 2009. Mapeamento da cobertura vegetal natural e antrópica do bioma Cerrado por meio de imagens Landsat ETM+. In Anais XIV Simpósio Brasileiro de Sensoriamento Remoto. Natal, p.1199-1206.

SANTOS, E.R., LOLIS, S.F., RODRIGUES, L.K.M. \& CARVALHO, Z.C. 2006. A flora do campus de Porto Nacional, Universidade Federal do Tocantins, Porto Nacional, Tocantins, Brasil. Rev. Ciênc. Agroambien. 1(1):61-67.

SANTOS, T.R.R., PINTO, J.R.R., LENZA, E. \& MEWS, H.A. 2012. The tree-shrub vegetation in rocky outcrop cerrado areas in Goiás State, Brazil. Braz. J. Bot. 35(3):281-294. http://dx.doi.org/10.1590/S180699592012000300007

SILVA, J.F., FARIÑAS, M.R., FELFILI, J.M. \& KLINK, C.A. 2006. Spatial heterogeneity, land use and conservation in the cerrado region of Brazil. J. Biogeog. 33(3):336-354. http://dx.doi.org/10.1111/j.13652699.2005.01422.x

TOCANTINS. Secretaria do Planejamento e da Modernização da Gestão Pública - SEPLAN. 2012. Atlas do Tocantins: subsídios ao planejamento da gestão territorial. 6. ed. SEPLAN, Palmas, 80p.

TÓTHMÉRÉSZ, B. 1995. Comparison of different methods for diversity ordering. J. Veg. Sci. 6(2):283-290. http://dx.doi.org/10.2307/3236223

WARMING, E. 1973. Lagoa Santa. In Lagoa Santa: a vegetação dos cerrados brasileiros (E. Warming \& M.G. Ferri, eds.). EDUSP, Itatiaia, São Paulo, Belo Horizonte, p.1-284.

ZAR, J.H. 1999. Biostatistical analysis. Prentice-Hall, New Jersey. 